\title{
PHASE TRANSITIONS IN ERBIUM-DOPED SILICON EXPOSED TO LASER RADIATION
}

\author{
R. I. Batalov, ${ }^{a}$ R. M. Bayazitov, ${ }^{\text {a }}$ D. I. Kryzhkov, \\ P. I. Gajduk, ${ }^{\text {c E. I. Gatskevich, }},{ }^{\text {G. }}$. D. Ivlev, ${ }^{*}$ \\ C. P. Marques, ${ }^{\mathrm{e}}$ and E. Alves ${ }^{\mathrm{e}}$
}

UDC 535.211;621.315.592

The dynamics of phase transitions induced by nanopulsed ruby laser radiation $\left(80 \mathrm{nsec}, 2 \mathrm{~J} / \mathrm{cm}^{2}\right)$ both in silicon layers doped with erbium ions and in those containing doped erbium and oxygen have been studied by an optical probing method. It is shown that the reflectivity behavior of structures under pulsed irradiation is governed by phase transitions (melting and crystallization) of implanted silicon and also by interference effects at the interfaces of the resulting phases. It is established that the profiles of erbium distribution change under nanosecond laser irradiation and that the dopant is forced out to the surface due to a segregation effect at small implantation doses. As the implanatation dose increases, diffusion deep into the sample tends to prevail over segregation. A considerable increase in the photoluminescence peak intensity at $0.81 \mathrm{eV}$ is found after both the pulsed laser processing and thermal post-annealing of doped samples as opposed to spectra of samples subjected either to thermal annealing or to pulsed laser irradiation.

Key words: doped silicon, erbium, ruby laser, reflectivity, phase transformations.

Introduction. Single-crystalline silicon is widely used in microelectronics to produce integrated circuits and is a non-direct-band semiconductor. Therefore, it is impractical for optoelectronics as a luminescent material. Doping silicon with erbium (Er) atoms is a promising way of creating instrument structures that emit in the communication range $\lambda=1.5-1.6 \mu \mathrm{m}$. Doping of $\mathrm{Er}^{+}$ions with subsequent high-temperature thermal annealing is the most common method of introducing rare-earth dopants into silicon. Silicon is additionally doped with oxygen ions in order to increase the solubility of $\mathrm{Er}$ in $\mathrm{Si}$ and to increase the luminescence intensity of the ${ }^{4} I_{13 / 2} \rightarrow{ }^{4} I_{15.2}$ transition [1]. The use of nanosecond laser action on doping of Si layers with $\mathrm{Er}$ has been little studied as an alternative to traditional thermal annealing [2]. This prompted an investigation of this phenomenon. Herein we report an in situ study of the dynamics of phase transitions (melting and crystallization) during irradiation of samples with nanosecond pulses of laser radiation and the diffusive redistribution of the dopant, the structural state, and the photoluminescence of the modified layers connected with these transitions.

Experimental. Single-crystalline wafers of $n$-type $\mathrm{Si}$ with the (100) orientation were doped at room temperature with $\mathrm{Er}^{+}$at energy $E=100 \mathrm{keV}$ at doses in the range $\Phi=10^{15}-10^{17} \mathrm{~cm}^{-2}$. Individual $\mathrm{Si}$ crystals were also doped with oxygen ions $\left(\mathrm{O}^{+}\right)$at energy $E=40 \mathrm{keV}$ at the fixed dose $\Phi=10^{17} \mathrm{~cm}^{-2}$. The width of the oxygen distribution profile at these ion energies was $d \approx 0.25 \mu \mathrm{m}$, the width of the erbium distribution was $d \approx 0.1 \mu \mathrm{m}$. Doped samples were subjected to pulsed laser treatment (PLT) in air. We used a ruby laser with a pulse length $\tau=80 \mathrm{nsec}$ and $\lambda_{\mathrm{em}}=0.69 \mu \mathrm{m}$. The optical path of the laser produced a highly homogeneous laser spot $[3,4]$. The unevenness of the energy distribution of a single pulse in the spot was less than $\pm 5 \%$. The irradiation energy was reproducible from pulse to pulse with a deviation of less than $\pm 1.5 \%$. The energy density of the incident radiation was $W=$ $2 \mathrm{~J} / \mathrm{cm}^{2}$.

*To whom correspondence should be addressed.

${ }^{a}$ Kazan Physical-Technical Institute, Russian Academy of Sciences; ${ }^{b}$ Institute of Physics of Microstructures, Russian Academy of Sciences, Nizhni Novgorod; ${ }^{c}$ Belarusian State University, Minsk; ${ }^{d}$ B. I. Stepanov Institute of Physics, National Academy of Sciences of Belarus, 22 Logojski Trakt, Minsk, 220090, Belarus; e-mail: ivlev@inel.basnet.by; ${ }^{\mathrm{I}}$ Instituto Tecnologico e Nuclear, Sacavem, Portugal. Translated from Zhurnal Prikladnoi Spektroskopii, Vol. 76, No. 2, pp. 225-231, March-April, 2009. Original article submitted September 2, 2008. 
The dynamics of laser-induced phase transitions during PLT were studied by detection of $p$-polarized probe radiation $(\lambda=1.06 \mu \mathrm{m})$ focused in a spot $(\approx 1 \mathrm{~mm})$ and directed at the sample at an angle of 40 that was reflected from the irradiated area. A sample of undoped $\mathrm{Si}$ was irradiated and the probe radiation reflection coefficient as a function of time was recorded for comparison.

The microstructure and phase composition of doped Si layers before and after PLT were studied by transmission electron microscopy (TEM) of samples prepared as planar cross sections on a Philips CM20 instrument. The distribution of Er atoms in Si before and after PLT was studied by Rutherford back-scattering using a beam of He ions at energy $E=2 \mathrm{MeV}$. Photoluminescence (PL) of samples was studied in the near-IR range $(1.0-1.7 \mu \mathrm{m})$ at $77 \mathrm{~K}$. The PL signal was produced by continuous irradiation with an Ar laser $(\lambda=514.5 \mathrm{~nm})$ at power $P=200 \mathrm{~mW}$. PL spectra were recorded using a BOMEM Fourier-spectrometer equipped with a cooled Ge radiation detector.

Results and Discussion. The sequence of laser-induced phase transitions can be seen in the dynamics of the reflectivity of doped Si subjected to PLT because the amorphous, crystalline, and liquid states of this material have different reflection coefficients. Figure 1 shows the active laser pulse and the reflectivity dynamics $R$ of single-crystalline $\mathrm{Si}$ and $\mathrm{Si}$ doped with Er. The initial values of the reflectivity coefficient of $p$-polarized probe radiation at this angle of incidence were estimated at 30-40\% depending on the sample type. For $W=2 \mathrm{~J} / \mathrm{cm}^{2}$, the duration of crystal-melt phase transitions in starting $\mathrm{Si}$ was determined by the temporal instability $\tau=200 \mathrm{nsec}$ of the reflectivity coefficient $R$. The increase of $R$ was due to Si melting; the plateau of $R$, reflection of probe radiation from the melted layer; a decrease of $R$, to the end of epitaxial crystallization (Fig. 1b). For laser radiation with the same $W$ on Si samples doped with $\mathrm{Er}$ at doses $\Phi=10^{15}$ and $10^{16} \mathrm{~cm}^{-2}$, the duration of the phase transitions changed very little. Si is known to become amorphous for $\Phi>10^{15} \mathrm{~cm}^{-2}$. A discontinuity related to intermediate crystallization [5] was observed for the minimal Er dose (Fig. 1c) at the front of $R$ growth as a result of the formation of a strongly overcooled $\mathrm{Si}$ melt at the reduced melting point $(\sim 200 \mathrm{~K})$ of the amorphous phase. The discontinuity in the increase of $R$ was practically invisible for $\Phi=10^{16} \mathrm{~cm}^{-2}$ (Fig. 1d). This may have been due to the rather high Er concentration $(N \approx$ $2 \cdot 10^{21} \mathrm{~cm}^{-3}$ ) that decreased the melt overcooling.

On going to the maximum dose $\left(\Phi=10^{17} \mathrm{~cm}^{-2}\right)$, the function $R(t)$ was qualitatively the same. The time $\tau$ increased to $300 \mathrm{nsec}$. Si began to melt slightly earlier. At first $R$ increased smoothly to a maximum and then fell below the initial level due to the hardening of the melted layer. The earlier melting may have been due to the fact that amorphous precipitates of Er silicides, the melting points of which were lower than amorphous $\mathrm{Si}$ were formed during ion doping. At a dose $\Phi=10^{17} \mathrm{~cm}^{-2}$, the Er atom concentration was $N \approx 10^{22} \mathrm{~cm}^{-3}$ and approached that of Si atoms $N \approx 5 \cdot 10^{22} \mathrm{~cm}^{-3}$. This corresponded to 20 at\% Er. According to the phase diagram of the Er-Si system [6], a metal-deficient $\mathrm{Er}$ disilicide $\left(T_{\text {melt }} \approx 1200^{\circ} \mathrm{C}\right)$ formed at this Er content. Precipitates of high-temperature silicides, e.g., ErSi with $T_{\text {melt }}=1900^{\circ} \mathrm{C}$, also formed during doping. This led to a smooth increase of $R$. The increase the melt lifetime to $300 \mathrm{nsec}$ could not have been related to segregation effects. A melt strongly enriched with Er was formed after the hardening was complete. This reduced the crystallization temperature and, therefore, increased the melt lifetime.

The action of the laser pulse on Si samples doped with $\mathrm{Er}$ and $\mathrm{O}$ typically gave a more complicated function $R(t)$ (Fig. 2). The presence of oxygen caused $\mathrm{Si}$ oxide to form. Er oxide also formed as the dose of doped $\mathrm{Er}^{+}$increased. The formation history of the composite doped layer [sequence of element doping: Er at various doses $(\Phi=$ $\left.10^{15}-10^{17} \mathrm{~cm}^{-2}\right)$ and $\mathrm{O}$ at a fixed dose $\left(10^{17} \mathrm{~cm}^{-2}\right)$ ] had a substantial effect on the dynamics of the dynamics of laser-induced transitions and $R(t)$. This can be seen by comparing oscillograms from samples with Er doping doses $10^{15} \mathrm{~cm}^{-2}$ (Fig. 2b and e). In the first instance, the reflectivity decreased after reaching the maximum $R$ to about the starting level and then decreased further (by $\sim 2$ times) after a slight increase when the melted layer was hardened. In the second instance, it decreased significantly after reaching the maximum compared with the starting value. The duration of the phase transitions in this instance was $\approx 100 \mathrm{nsec}$ longer. The difference in the dynamics of $R(t)$ can be seen from oscillograms for samples with Er doping doses $\Phi \approx 10^{16} \mathrm{~cm}^{-2}$ (Fig. 2c and f).

The melt formed by laser action on a Si:Er layer exhibited properties of a liquid metal with Er concentration from 0.2 at $\%\left(\Phi=10^{15} \mathrm{~cm}^{-2}\right)$ to 20 at $\%\left(10^{17} \mathrm{~cm}^{-2}\right)$. The reflectivity of the probe radiation from the sample when the thickness of the melted layer was greater than the thickness of the skin layer, the typical value of which was of the order of $10 \mathrm{~nm}$, was determined by the optical parameters of just this melt. A system with layers having different optical properties was probably generated from the start and further development of phase transitions from the action of laser pulses on samples with a high Er and $\mathrm{O}$ atom concentrations $\left(\Phi=10^{17} \mathrm{~cm}^{-2}\right.$, which corresponded to bulk 


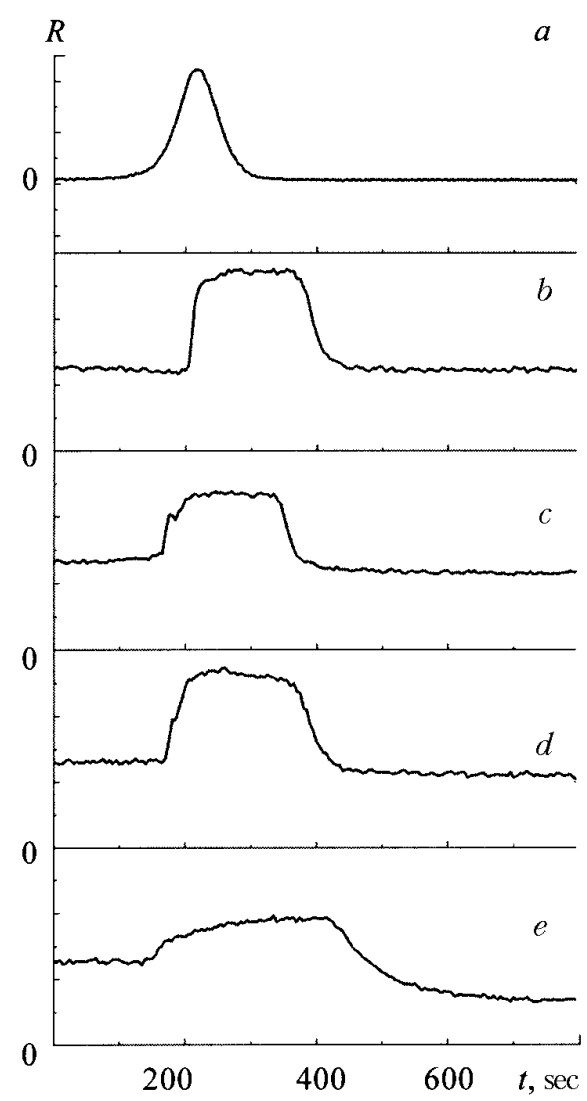

Fig. 1. Laser pulse $\left(\tau=80 \mathrm{nsec}, W=2 \mathrm{~J} / \mathrm{cm}^{2}\right)$ (a) and change of reflectivity caused by it at $\lambda=1.06 \mu \mathrm{m}$ in samples of starting single-crystal $\mathrm{Si}(\mathrm{b})$ and $\mathrm{Si}$ doped with Er at doses $\Phi=10^{15}(\mathrm{c}), 10^{16}(\mathrm{~d})$, and $10^{17} \mathrm{~cm}^{-2}(\mathrm{e})$. The $x$ axis corresponds to level $R=0$ (b-e).

concentration $N \approx 10^{22} \mathrm{~cm}^{-2}$ ). As a result, interference effects contributed to the dynamics of $R(t)$ upon reflection of the probe radiation. Interference changing with time can explain the path of $R(t)$ for the sample doped with $\mathrm{Er}^{+}$at dose $\Phi=10^{16} \mathrm{~cm}^{-2}$ upon laser irradiation (Fig. 2c). The results for the reflectivity of the samples can be partially explained based on structural studies of the layers and the distribution of Er dopant along the depth profile.

Figure 3 shows typical Rutherford back-scattering spectra for an unoriented beam of He ions that were measured on Si doped with Er and O before and after PLT. Vertical arrows indicate the energies of particles scattered on surface $\mathrm{Si}$ and $\mathrm{Er}$ atoms. Distribution profiles of $\mathrm{Si}$ (energy range 1.2-1.5 MeV) and $\mathrm{Er}(1.8-2.0 \mathrm{MeV}$ ) after ion doping of $\mathrm{O}$ and Er can be derived from curve 1. The profile of the Er distribution was almost Gaussian. This was consistent with a small effect on the path of Er ions from a starting $\mathrm{Si}$ matrix doped with $\mathrm{O}$. The decrease in the $\mathrm{Si}$ spectrum at energy $1.4 \mathrm{MeV}$ corresponded to the maximum distribution of Er atoms that was $\approx 50 \mathrm{~nm}$ according to the SRIM-2006 program [7].

The Er redistributed as a result of the PLT (Fig. 3). Part of the Er atoms migrated to the surface (a narrow peak of width $\approx 20 \mathrm{~nm}$ was observed in the concentration profile). The majority of the atoms diffused deep into the sample. The Rutherford back-scattering spectrum of $\mathrm{Si}$ showed a maximum at $1.4 \mathrm{MeV}$ that was directly related to a minimum in the Er spectrum at $1.9 \mathrm{MeV}$. Part of the Er dopant migrated to the surface (segregated) because of the low solubility of $\mathrm{Er}$ in $\mathrm{Si}\left(\sim 10^{16} \mathrm{~cm}^{-3}\right)$. The small width was a consequency of the high rate $(\sim 1 \mathrm{~m} / \mathrm{sec})$ of movement of the crystallization front during hardening of the Si melt when practically the whole dopant migrated as a thin subsurface layer up to $30 \mathrm{~nm}$ thick. The dopant concentration in the melt increased as the doping dose increased. The $\mathrm{Si}-\mathrm{Er}$ melt composition approached the eutectic value. Spectrum 2 showed two peaks for the Er concentration. One near the surface was due to segregation phenomena; a second peak, to diffusion of Er into the sample. Diffusion into 


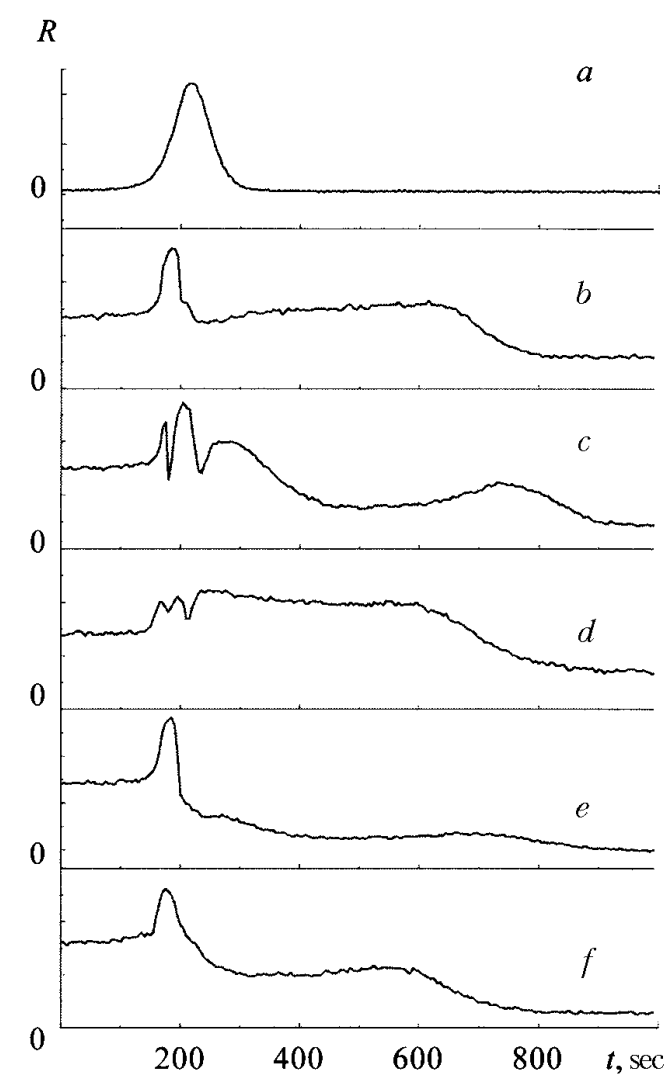

Fig. 2. Laser pulse $\left(\tau=80 \mathrm{nsec}, W=2 \mathrm{~J} / \mathrm{cm}^{2}\right)$ (a) and change of reflectivity caused by it at $\lambda=1.06 \mu \mathrm{m}$ in samples of $\mathrm{Si}$ doped with oxygen $(E=40$ $\left.\mathrm{keV}, \Phi=10^{17} \mathrm{~cm}^{-2}\right)$ and Er ions $(E=100 \mathrm{keV})$ at various doses $\Phi=10^{15}$ (b), $10^{16}(\mathrm{c})$, and $10^{17}(\mathrm{~d}), 10^{15}(\mathrm{e})$, and $1.6 \cdot 10^{16} \mathrm{~cm}^{-2}(\mathrm{f})$. The $x$ axis corresponds to level $R=0$ (b-f).

the material began to prevail over segregation onto the surface as the Er concentration increased. We observed similar redistribution of $\mathrm{Fe}$ in $\mathrm{Si}[8]$ during PLT.

Rutherford back-scattering spectra for an oriented beam of He ions (not shown) indicated, firstly, that the width of the disordered $\mathrm{Si}$ region after $\mathrm{Er}$ and $\mathrm{O}$ doping was $\approx 0.2 \mu \mathrm{m}$, which was comparable with the width of the O distribution profile $(\approx 180 \mathrm{~nm})$ [7]; secondly, that Er atoms after PLT were located in interstices in the Si lattice; and, thirdly, that PLT produced epitaxial crystallization of doped Si layers with a high concentration $\left(\sim 10^{22} \mathrm{~cm}^{-3}\right)$ of Er and $\mathrm{O}$.

The microstructure of the doped Si layers before and after PLT was investigated using TEM. According to the results, it typically had before PLT a continuous amorphous layer with embedded particles ( $\mathrm{Si}$ and silicides of Er) of dimensions up to $30 \mathrm{~nm}$ without clear boundaries. The microdiffraction pattern of this layer gave strongly broadened rings from Si. The light-field TEM image of the doped Si layer after PLT (Fig. 4) indicated formation of the cellular structure typical for liquid epitaxy of highly supersaturated alloys. According to dark-field and diffraction measurements, this was single-crystalline cells (pillars) of $\mathrm{Si}$ of dimensions $40-80 \mathrm{~nm}$ directed toward the surface and separated by walls in which the Er dopant was primarily concentrated as single-crystalline $\mathrm{ErSi}_{2}$. The single-crystalline structure of $\mathrm{Si}$ and $\mathrm{ErSi}_{2}$ in the cellular structure was inferred from point reflections on microdiffraction photographs and was confirmed by x-ray diffraction spectra, in which only one peak of $\mathrm{ErSi}_{2}$ (101) was observed. A study of the microstructure of Si layers after PLT and additional thermal annealing at $T=800-900^{\circ} \mathrm{C}$ showed that the cellular structure was thermally stable. 


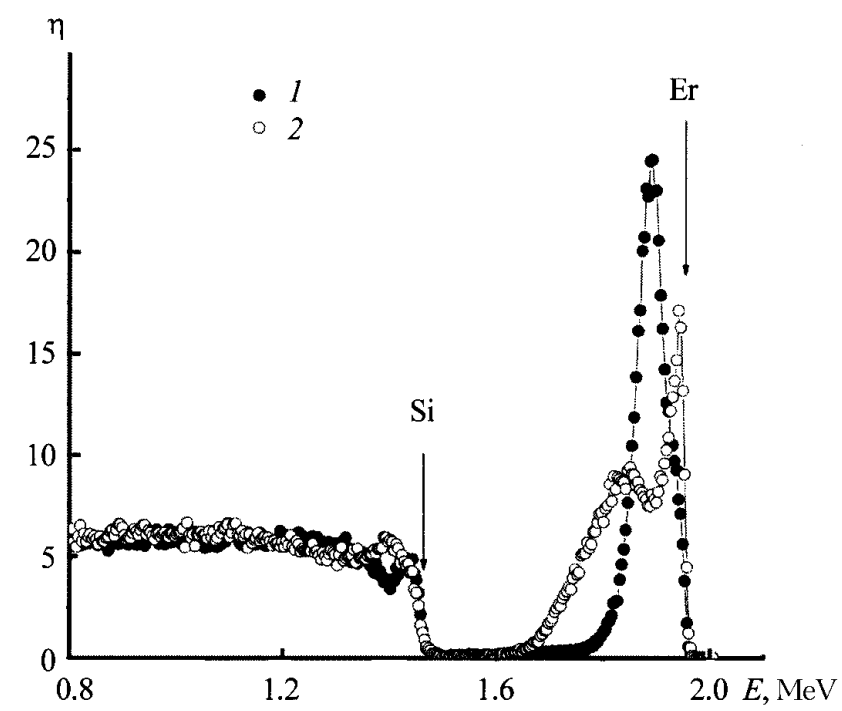

Fig. 3. Rutherford back-scattering spectra of an unoriented beam of He ions measured for Si doped with oxygen $\left(E=40 \mathrm{keV}, \Phi=10^{17} \mathrm{~cm}^{-2}\right)$ and $\mathrm{Er}$ $\left(E=100 \mathrm{keV}, \Phi=6 \cdot 10^{16} \mathrm{~cm}^{-2}\right)$ before (1) and after (2) pulsed laser treatment $\left(\tau=80 \mathrm{nsec}, W=2 \mathrm{~J} / \mathrm{cm}^{2}\right)$. $\eta$ is the normalized yield; arrows indicate energies of particles scattered at $\mathrm{Si}$ and Er surface atoms.

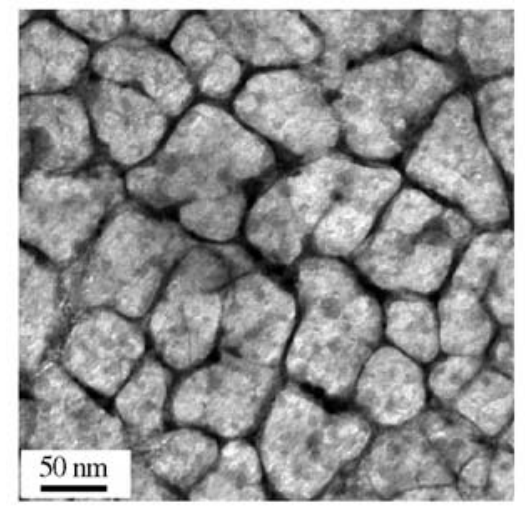

Fig. 4. TEM image of Si layer doped with oxygen $\left(E=40 \mathrm{keV}, \Phi=10^{17}\right.$ $\left.\mathrm{cm}^{-2}\right)$ and $\operatorname{Er}\left(100 \mathrm{keV}, 6 \cdot 10^{16} \mathrm{~cm}^{-2}\right)$ after pulsed laser treatment $(\tau=80$ msec, $\left.W=2 \mathrm{~J} / \mathrm{cm}^{2}\right)$.

PL spectra in the near-IR range were measured on samples doped with $\mathrm{O}$ at a dose of $10^{17} \mathrm{~cm}^{-2}$ and with Er at doses $10^{15}-10^{17} \mathrm{~cm}^{-2}$. Measurements of PL in doped layers after PLT did not reveal any visible PL peaks in the range 1.0-1.7 $\mu \mathrm{m}$ that could result from an increased concentration of point defects formed as the result of rapid crystallization from the melt and acting as centers of radiationless recombination. Peaks with maxima at $\approx 0.81$ and $\approx 1.10 \mathrm{eV}$ that were due to transitions between $\mathrm{Er}^{4} I_{13 / 2} \rightarrow{ }^{4} I_{15 / 2}$ levels and edge luminescence of Siappeared in $\mathrm{Pl}$ spectra after brief thermal annealing $\left(800^{\circ} \mathrm{C}, 20 \mathrm{~min}\right)$ to remove these defects. Figure 5 shows PL spectra of a sample doped with the lowest Er dose $\left(\Phi=10^{15} \mathrm{~cm}^{-2}\right)$. It can be seen that PLT with subsequent high-temperature annealing produced a much (up to five times) stronger PL peak from Er at $\approx 0.81 \mathrm{eV}$ compared with ordinary thermal annealing. This difference is related to the fact that the layer converted to the crystalline state as a result of laser-induced melting and subsequent hardening of the doped layer whereas a polycrystalline layer with an increased number of defects was formed after ordinary thermal annealing. It was also established that the intensity of the Er peak decreased on increasing the Er dose from $10^{16} \mathrm{~cm}^{-2}$ to $10^{17} \mathrm{~cm}^{-2}$ whereas the intensity of the Si PL peak increased and became saturated. 


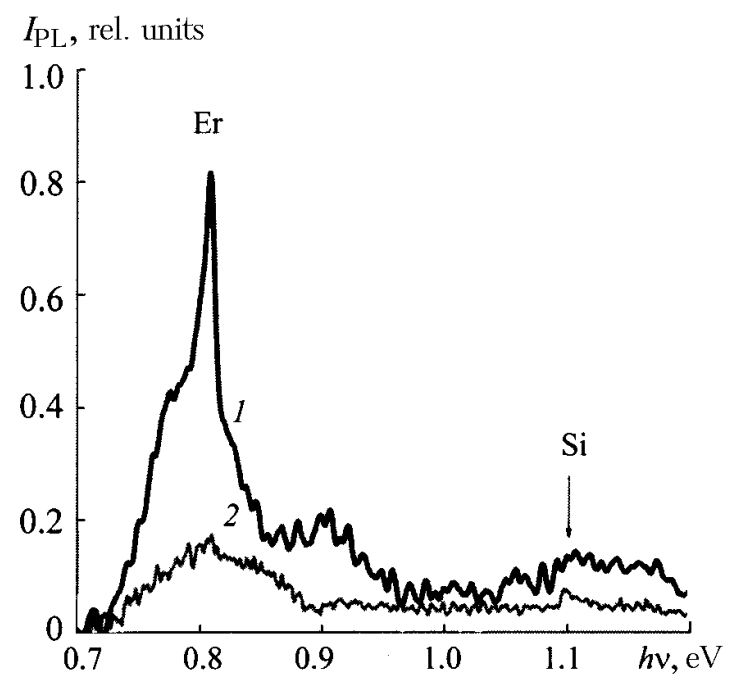

Fig. 5. Photoluminscence spectra $(T=77 \mathrm{~K})$ of a $\mathrm{Si}$ layer doped with oxygen $\left(E=40 \mathrm{keV}, \Phi=10^{17}\right)$ and $\operatorname{Er}\left(100 \mathrm{keV}, 10^{15} \mathrm{~cm}^{-2}\right)$ after pulsed laser treatment $\left(W=2 \mathrm{~J} / \mathrm{cm}^{2}\right)$ and high-temperature annealing $\left(800{ }^{\circ} \mathrm{C}, 20 \mathrm{~min}\right)(1)$ and after high-temperature annealing without pulsed laser treatment (2).

The weakening of the Er peak was related to its precipitation into optically inactive metallic Er slicides whereas the strengthening of the Si peak was due to formation of single-crystalline cellular structures (Fig. 4) at doses close to $10^{16} \mathrm{~cm}^{-2}$ that were not observed at a dose of $10^{15} \mathrm{~cm}^{-2}$. Thus, nanosecond laser action with subsequent high-temperature annealing increased the PL intensity, indicating that the crystalline structure of the doped layers was restored.

Conclusion. The dynamics of the action of nanopulse $\left(80 \mathrm{~nm}, 2 \mathrm{~J} / \mathrm{cm}^{2}\right)$ radiation from a ruby laser on samples of Si doped with $\mathrm{Er}$ and $\mathrm{Si}$ doped with $\mathrm{Er}$ and $\mathrm{O}$ was studied by optical probing. Features of the reflectivity dynamics of the various samples under conditions leading to laser-induced phase transitions that produced a new state of surface layers were established. It was shown that nanosecond laser action changes the distribution of doped Er. The dopant migrated to the surface at low doses as the result of a segregation effect whereas diffusion of $\mathrm{Er}^{+}$deep into the sample during PLT was observed at increased dopant doses and began to prevail over segregation. The Er PL peak intensity at $0.81 \mathrm{eV}$ increased significantly after PLT and subsequent annealing of the doped samples when compared with samples subjected to only thermal annealing or only PLT.

Acknowledgments. The work was supported partially by the State Complex Program of Scientific Research Electronics (Electronics 4.10), the Russian Foundation for Basic Research (Grant No. 08-02-01280) of the Program of the Division of Physical Sciences of the Russian Academy of Sciences New Materials and Structures, and the Foundation for Cooperation in Domestic Science.

\section{REFERENCES}

1. A. Polman, J. Appl. Phys., 82, 1-39 (1997).

2. J. Michel, J. L. Benton, R. F. Ferrante, D. C. Jacobson, D. J. Eaglesham, E. A .Fitzgerald, Y.-H. Xie, J. M. Poate, and L. C. Kimerling, J. Appl. Phys., 70, 2671-2678 (1991).

3. G. D. Ivlev and V. L. Malevich, Kvantovaya Élektron., 15, 2584-2586 (1988).

4. E. I. Gatskevich, G. D. Ivlev, and A. M. Chaplanov, Kvantovaya Élektron., 22, 805-810 (1995).

5. G. D. Ivlev, Zh. Tekh. Fiz., 61, 195-197 (1991).

6. S. P. Luzan, V. E. Listovnichii, Yu. I. Buyanov, and P. S. Martsenyuk, J. Alloys Cmpd., 239, 77-82 (1996).

7. J. F. Ziegler, M. D. Ziegler, and J. P. Biersack, The Stopping and Range of Ions in Matter, SRIM-2006 Computer Program, http://www.srim.org.

8. R. M. Bayazitov, R. I. Batalov, I. B. Khaibullin, G. D. Ivlev, I. Dezsi, and E. Kotai, J. Phys. D: Appl. Phys., 37, 468-471 (2004). 